\title{
Carnets
}

Revue électronique d'études françaises de l'APEF

Deuxième série - $10 \mid 2017$

Pratiques de l'errance, vécus de la mémoire

\section{Paysage, chemin et errance chez Julien Gracq}

\section{Ana Isabel Moniz}

\section{(2) OpenEdition}

Journals

Édition électronique

URL : http://journals.openedition.org/carnets/2260

DOI : $10.4000 /$ carnets. 2260

ISSN : 1646-7698

Éditeur

APEF

Référence électronique

Ana Isabel Moniz, «Paysage, chemin et errance chez Julien Gracq », Carnets [En ligne], Deuxième série - 10 | 2017, mis en ligne le 30 avril 2017, consulté le 10 décembre 2020. URL : http:// journals.openedition.org/carnets/2260 ; DOI : https://doi.org/10.4000/carnets.2260

Ce document a été généré automatiquement le 10 décembre 2020.

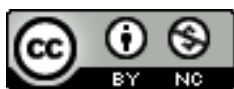

Carnets est mis à disposition selon les termes de la licence Creative Commons - Atribution - Pas d'utilisation commerciale 4.0 International. 


\title{
Paysage, chemin et errance chez Julien Gracq
}

\author{
Ana Isabel Moniz
}

1 Voyager, aller, se déplacer, vagabonder, partir en quête, peut être appréhendé comme un mouvement représenté par une traversée dans l'espace géographique. ${ }^{1}$ Depuis le témoignage de la Bible, avec la traversée du désert par Abraham, jusqu'à nos jours, dont peuvent être un cas les flux de migrants, la littérature est témoin d'innombrables exemples de déplacements dans l'espace qui, de leur côté, se déploient en voyages intérieurs. Une modalité de l'errance menée par les pensées, les rêveries qui permettent au sujet de dépasser ses limites et ainsi les bornes du réel pour accéder à l'inconnu.

2 L'errance est une thématique qui se répète constamment dans l'œuvre de Julien Gracq. Elle postule une inclinaison particulière pour affronter de nouvelles réalités qui peuvent aller à la rencontre d'un profond souhait de changement intérieur. Du point de vue de la création littéraire, l'errance est un sujet qui, généralement, traduit une énorme insatisfaction chez le héros, obligeant ce dernier à se mettre en quête de nouvelles perspectives. Comme rompement avec l'autre pour une transformation drastique de sa vie au travers de successives infractions, l'errance est aussi bien dangereuse pour l'identité que pour l'intégrité d'un sujet qui s'efface pour réapparaitre, transformé et transcendé.

3 Je me propose d'analyser cette problématique dans l'univers fictionnel de Julien Gracq, surtout à partir d'Un Balcon en forêt, un récit de guerre à la troisième personne, publié en 1958. Dans ce livre, la réalisation du voyage/de l'errance qui coïncide avec le début du parcours textuel suggère une rupture du héros avec le passé et qui, mobilisé, devra partir à la guerre. Son itinéraire, paradoxalement de libération, lui permettra non seulement de connaitre Mona et l'amour, mais surtout d'essayer de se retrouver et de se reconnaître par le biais de l'expérience de retour à l'origine de sa vie, en pleine communion avec la nature.

4 Envoyé par son colonel dans la forêt de Falizes, isolé au sommet d'une montagne, «il bénissait ce mauvais chemin qui lui faisait les coudées franches et coupait le Toit à demi du monde habité » (Gracq, 1995a : 21). Loin de tout et de tous, il contemple le monde et 
l'agitation provoquée par la "drôle de guerre» pendant l'hiver 1939-1940. Lors des affrontements, la coupe des communications avec la ville conduit le protagoniste et ses collègues de la «maison-fort » à entamer d'autres parcours totalement étrangers au devoir militaire. La guerre assume cependant une dimension plus grave lorsque les bombardements des Allemands laissent Aldo, le héros, isolé et livré à lui-même, dans une forêt qui ne le protégera pas : «Ce voyage à travers la forêt cloîtrée par la brume poussait Grange peu à peu sur la pente de sa rêverie préférée ; il y voyait l'image de sa vie : tout ce qu'il avait, il le portait avec lui » (Gracq, 1995a: 26).

5 Les déplacements successifs dans l'espace qui sépare la ville de Moriarmé de la forêt de Falizes, où se situe la maison-fort, renvoient à la thématique du lieu élevé, dans sa représentation symbolique d'un monde distant et inconnu. Dans ce même sens, le transfert de Grange en "Toit », désignation attribuée à la maison-fort où il doit maintenir sa fonction de surveillance, renvoie à cette autre réalité, inconnue et inaccessible, par le biais de la métaphore et du lieu élevé gravé dans le nom. Le parcours d'ascension qui conduit le héros dans ce lieu l'oblige à s'écarter des lieux communs, à peine guidé par le vertige causé par les hauteurs, dans une constante attraction vers l'abîme, le néant, la mort.

6 La forêt qu'il doit traverser et l'espace particulier de l'errance, en accord avec la configuration imaginaire que le processus initiatique assume chez Gracq, se présente sombre et silencieuse, déjà dans son engourdissement de mort, à l'image de celles qui, dans d'autres textes de Gracq, s'imposent aux protagonistes :

Jamais Grange n'avait eu comme ce soir le sentiment d'habiter une forêt perdue : toute l'immensité de l'Ardenne respirait dans cette clairière de fantômes, comme le cœur d'une forêt magique palpite autour de sa fontaine. Ce vide de la futaie, cette garde sommeillante le troublaient. Il songeait au mot bizarre (...) d'Hervouët : « on n'est pas soutenus ». Ce qu'on avait laissé derrière soi, ce qu'on était censé défendre, n'importait plus très réellement; le lien était coupé ; dans cette obscurité pleine de pressentiments les raisons d'être avaient perdu leurs dents (Gracq, 1995a: 86-87).

Ce néant est considéré par Simone Grossman comme un signe qui révèle une vie secrète et invisible. Si la forêt semble toujours marquer la frontière avec le monde interdit, la mer, cette "entité légendaire douée d'une puissance d'envoûtement » (Grossman, 1980 : 164), est aussi un lieu séduisant qui a la même fonction : celle de séparer la vision du réel de celle du rêve. Inséparables, ces deux espaces semblent s'unir pour répondre au défi de l'approximation de l'homme avec le mystère, passant de l'un à l'autre au travers d'une frontière naturelle. Mais il est possible que la mer ne soit suggérée que dans le contraste de la zone illuminée de la forêt avec son obscurité : «L'obscurité de la forêt où cahotait cette petite lumière en paraissait soudain plus vaste, plus douteuse, confuse et égarante comme la mer » (Gracq, 1995a : 104).

De cette façon, le liquide et le végétal viennent représenter l'atmosphère propice à l'aventure de ces héros, "[qui] n'habitent jamais chez eux» et qui ont comme « résidences secondaires : mer et forêt» (Gracq, 1995b: 153). Nous connaissons, par le biais de Gilbert Durand, la charge symbolique que ces espaces possèdent : la forêt comme espace sacré et centre d'intimité, la mer par sa profondeur, archétype de la descente et du retour à l'origine de la vie et de la félicité originelle, étant donné la liaison entre l'élément liquide et la naissance.

9 Forêt et mer deviennent ainsi la localisation idéale pour l'évènement. «La forêt, c'est l'endroit où se produit l'apparition » (Ernst, $1997: 220)$, a dit l'auteur dans une entrevue 
concédée à Gilbert Ernst, pendant laquelle il a aussi justifié son affirmation en s'appuyant, pour cela, sur les légendes médiévales : "C'est toujours dans la forêt que se situent dans les légendes du Moyen Âge les apparitions, les événements, le miraculeux. Tout peut apparaître dans la forêt, c'est par excellence le lieu mystérieux, magique » (Ernst, 1997 : 220-221).

C'est pourquoi la constante errance par ces lieux s'impose comme itinéraires indispensables au parcours de l'existence et de l'aventure des entités fictionnelles qui réagissent cependant devant son attraction de façon contradictoire. Comme espaces limite de la représentation de l'absence, toujours silencieux et vides, ils tendent à s'imposer, dans la pratique fictionnelle de Gracq, en corrélation avec l'errance, l'attente et la mort.

11 Dans les écrits de Gracq, la peur et le désir semblent susciter la même relation des personnages avec ces espaces mystérieux et magiques. Si la forêt fonctionne doublement, motivant l'attraction et la répulsion, c'est aussi parce qu'elle se dessine comme un lieu irréel de «bois pleins de bêtes et de surprises » (Gracq, 1995a : 7), comme cela est décrit dans Un balcon en forêt.

12 Par son aspect obscur, l'errance dans la forêt éveille l'inquiétude chez qui s'y aventure, révélant la menace des dangers qu'elle a l'air de dissimuler. Obéissant à une impulsion qui semble l'orienter, le héros se laissera séduire par ce lieu qui cache cependant le tragique destin qui l'attend lorsque, postérieurement, il se transforme en lieu de mort.

Bien que quelque peu semblable à Storrvan par son épaisseur menaçante, forêt " confuse et écartée » (Gracq, 1989a: 10) dans laquelle se situe le château d'Argol et l'action du premier roman de Gracq, publié en 1938, la forêt des Ardennes d'Un balcon en forêt, « sans une déchirure, sans une clairière » (Gracq, 1995a : 8), semble plus conviviale et favorable aux incursions. En cet endroit, l'obscurité n'est pas aussi totale que l'auteur prétend nous le faire croire par le biais du narrateur : " Il regardait monter lentement au-dessus de la forêt une grosse lune blême; sur le chemin qu'elle éclairait obliquement, la coulée de pierres râpeuse se hérissait d'ombres coupantes, redevenait un lit de torrent » (Gracq, 1995a : 118).

14 Cette dualité, représentée dans la fiction par les jeux d'ombre et de clarté, finit par se déployer dans l'amalgame de sentiments contradictoires vécus par les entités fictionnelles. Oscillant entre l'angoisse et le désir, le héros cherche à passer de l'obscurité mystérieuse à la clarté révélatrice, un désir qui l'a toujours accompagné dans ses parcours d'aventure. Grange cherche dans la forêt la solitude qui le mènera à la rencontre de la révélation transcendante au « cœur du profond remue-ménage de la terre »:

rien ne lui semblait plus importer que d'être assis sur le bord de ce torrent, au cœur du profond remue-ménage de la terre. Il sentait bien au creux du ventre une révulsion désagréable (...); il comprenait que c'était la peur d'être tué; mais une part en lui se détachait et flottait au fil de la nuit légère : il éprouvait quelque chose de ce que durent ressentir les passagers de l'arche, lorsque les eaux commencèrent à la soulever (Gracq, 1995a : 118).

15 Comme espace privilégié par la narration fictionnelle de Gracq, la forêt reçoit aussi la valorisation d'un être animé. En s'humanisant, elle fait du projet de vie des héros son propre projet de vie, tout en s'identifiant avec eux. Dans cette solidarité, elle semble assumer cette posture frappante, de surveillant attentif, puisqu'elle se détache, dans le paysage « debout et immobile comme un homme » (Gracq, 1995a : 163). 
16 Sur la base d'une confrontation infinie avec le destin, son parcours de vie est, lui aussi, marqué par des moments de profonde obscurité. Le narrateur d'Un balcon en forêt souhaite le souligner lorsqu'il affirme qu': « Une idée bizarre se glissait dans l'esprit de Grange : il lui semblait qu'il marchait dans cette forêt insolite comme dans sa propre vie » (Gracq, 1995a: 83).

17 Représentation symbolique de la vie, la forêt, "à la ressemblance de la vie humaine ", vient s'imposer ici, selon la perspective de J. L. Leutrat, comme "un grand être qui écoute, qui respire, qui attend, qui croît («Il écoutait pousser la forêt») et qui est parcourue du frémissement des signes » (Leutrat, $1967: 68$ ).

18 L'animation de la nature, processus rhétorique auquel Gracq fait fréquemment appel, se présente de cette façon comme métaphore de l'homme, dans ses fonctions les plus élémentaires de respirer, sentir, (re)vivre :

La forêt respirait, plus ample, plus éveillée, attentive jusqu'au fond de ses forts et de ses caches soudain remués aux signes énigmatiques d'on ne savait quel retour des temps (...) on eût dit que la vieille bauge mérovingienne flairait encore dans l'air un parfum oublié qui la faisait revivre (Gracq, 1995a : 37).

19 Les héros de Gracq, désignés dans Le Rivage des Syrtes sous le nom de "poètes de l'événement » (Gracq, 1989c: 775) ont ainsi, comme objectif de parcours, celui de surpasser la réalité apparente du monde réel, de façon à atteindre son côté obscur: « Passer aussi de l'autre côté, éprouver à la fois la pesée et la résistance » (Gracq, 1989c : 775).

20 Le moment de la transgression arrivé, le livre se ferme sur une construction symbolique circulaire, toujours habitée par l'absence et répétant sans cesse la même attitude qui oriente les personnages dans leur tentative de surpasser les frontières de la supra-réalité.

21 Ainsi, dans la configuration de l'espace soumis à l'imaginaire de Gracq, l'errance dans l'espace, définie par Dominique Berthet comme un « long périple à la recherche de ce lieu acceptable dont l'inattendu, l'inconnu (...) sont les composantes » (Berthet, $2007: 11$ ), a tendance à se déployer en incursions à l'intérieur du personnage qui assume la narration, ce qui permet de révéler ses aspirations, déterminantes pour le développement de l'intrigue elle-même. Inhérent au parcours des héros, ce changement de lieu par le biais d'un ou de plusieurs déplacements, renvoie, symboliquement, vers un autre état spirituel, où il lui est possible de rapprocher ces personnages d'une autre réalité, supérieure. C'est dans ce sens que, poussés par ce désir qu'eux-mêmes ne savent expliquer, ils se fixent dans le cadre d'un autre paysage, distant de celui de leurs origines, assumant formes et sens multiples, ce qui les conduit à la dérive de l'imaginaire, au seuil qui sépare ces deux réalités. Leur drame est surtout basé sur le fait qu'ils ne peuvent vivre, simultanément, sur ces deux plans distincts. L'unique possibilité d'annuler l'ambiguïté génératrice de ce conflit semble être, en accord avec les prémisses surréalistes, dans la mort, le lieu ultime de l'existence qui permettrait la coexistence de ces deux plans.

C'est peut-être pour être prédestinés à errer que les héros de Gracq ne questionnent pas ce que leur propre construction les oblige à effectuer. C'est le cas de Grange qui, après avoir été mobilisé pour aller à la guerre, est forcé de quitter la ville de Moriarmé pour s'installer dans la maison-fort, dans la forêt de Falizes. Il constate la présence d' ' un autre monde, un monde silencieux et intimidant, baigné d'une lumière blanche, d'une évidence calme » (Gracq, 1995a: 17), d'où semble émaner un étrange sentiment de liberté insolite, manifesté par son affirmation: "Il n'y a que moi au monde, se disait-il avec une 
allégresse qui l'emportait. (...) Je suis seul ici. Je fais ce que je veux » (Gracq, 1995a : 52 et 113).

La distance qui sépare Moriarmé de Falizes, les limites géographiques des principales étapes d'Un balcon en forêt, semble avoir pour but de séparer la frontière entre le connu et l'inconnu. C'est dans ce nouveau paysage que le héros sent le plus intensément la présence d'un monde nouveau, encore à dévoiler, dans lequel il lui sera possible de s'approcher de la révélation, déjà préfigurée dans le silence "magique » qui entoure le lieu: "Le silence du lieu devenait alors presque magique. Un sentiment bizarre l'envahissait chaque fois qu'il allumait sa cigarette dans ce sous-bois perdu : il lui semblait qu'il larguait ses attaches; il entrait dans un monde racheté, lavé de l'homme » (Gracq, 1995a : 51-52).

La tendance pour l'errance comme objectif de se fixer dans d'autres lieux, fréquemment manifestée par les entités fictionnelles de Gracq, semble être le résultat de sa fascination pour des «mondes imaginaires», en accord avec Pierre Jourde, dotés d'une relative «ressemblance avec la réalité» (Jourde, 1991: 11). En tant qu'espace intériorisé, ils représentent la synthèse d'une présence spatiale et d'un projet intime. Essayer de le dévoiler dans ces deux versants correspond à la quête inhérente au parcours de chaque personnage. Laisser l'espace connu pour avancer vers l'autre, seulement pressenti et souhaité est une façon de répondre à cet appel intime, "appel impérieux (...) à la trouvaille » (Gracq, 1989b: 506), selon l'expression utilisée par Gracq dans son essai consacré à Breton.

Pour le héros, errer vers un lieu où il devra se trouver face à face avec la connaissance, implique se soumettre à diverses étapes, qui se dérouleront selon ses successifs déplacements et qui correspondront à un déchiffrement graduel de son parcours. En dehors de ces contours réels, ces espaces qu'il traversera éveilleront en lui le désir de concrétiser une quête intérieure, le poussant à interpréter et à déchiffrer les pistes et les signaux d'un monde interdit, lancés à mesure que son aventure se déroulera.

Parfois, les signaux deviennent des présages de la guerre ; en particulier dans Un balcon en forêt, lorsque Grange est dans une de ses missions de surveillance, dans la forêt des Ardennes qui semble, subitement, l'alerter sur l'imminence d'un danger: "La terre sourdement alertée était de nouveau pleine de présages (...) » (Gracq, 1995a : 53).

Le personnage gracquien concrétise, de cette façon, son parcours, toujours poussé par la fascination d'une possible révélation. Son errance le conduit à une confrontation avec des énergies surhumaines qui marque son itinéraire vers des chemins de transgression : dans Au château d'Argol, premier livre de Julien Gracq publié en 1938, la transgression finale, configurée par le suicide de Heide et par la mort d'Herminien, est précédée par d'autres, celle du viol de Heide par Herminien et, postérieurement, par Albert, ainsi que celle des limites de l'existence humaine à travers la tentative volontaire de noyade réalisée par le trio dans les mers d'Argol. Dans Un beau ténébreux, l'aventure de la découverte des énigmes que le voyage propose et que la narration accompagne, culmine avec le suicide du protagoniste et de sa compagne, une fois de plus, à travers la transgression des limites de la propre vie, c'est-à-dire, de la frontière qui sépare la vie de la mort, le réel du transcendant ; dans Le Rivage des Syrtes, la violation de la frontière qui sépare Orsenna du Farghestan et qui représente la réalisation du destin d'Aldo, mais aussi la libération d'un peuple; dans Un balcon en forêt, la transgression émerge sur le plan de la propre intériorité du héros qui, répondant à l'appel fondamental du "lâchez-tout " (Gracq, 1995a : 114), « ce besoin de faire sauter une à une les amarres » (Gracq, 1995a: 114), se 
distancie du monde et de sa réalité pour entrer dans une espèce de communion avec la nature, avec laquelle il finit par fusionner.

Pour Simone Grossman, il y a une liaison entre le mouvement surréaliste et le héros de Gracq. Celle-ci est basée sur le type d'errance et de quête développées dans les deux cas, qui consiste, précisément, en une transgression jusqu'aux limites normalement consenties par la dimension humaine. "La transgression n'est pas la négation de l'interdit mais elle le dépasse et le complète »(Bataille, 1974: 70), affirme Georges Bataille.

Cette transgression a sans doute pour objectif d'obliger le héros à pénétrer dans les domaines interdits à l'Homme pour, de cette façon, le situer en même temps des deux côtés de la frontière. En accord avec la conception surréaliste, elle viserait le point neutre de la rencontre du rêve et de la réalité. À travers la transposition des marges de cet interdit, le héros de Gracq aurait pour objectif d'atteindre l'idéal du mouvement orienté par Breton, « ce point de l'esprit d'où la vie et la mort, le réel et l'imaginaire, le passé et le futur, le communicable et l'incommunicable, le haut et le bas cessent d'être perçus contradictoirement » (Breton, $1988: 781$ ).

La retraite et l'isolement qui le façonnent le rendent différent des autres entités fictionnelles, dans la mesure où, séparé et distant des autres, il est l'élu, celui auquel est donnée la possibilité de se rapprocher de ce certain "point de l'esprit», permettant l'annulation des frontières entre le monde et le transcendant, entre le réel et la «surréalité ».

31 Dans ce sens, chez Julien Gracq, le mouvement essentiel du texte se concentre autour de l'errance et de la tension d'une attente intérieure qu'elle suscite, mais aussi autour des transgressions de limites et de frontières qu'il traverse dans la recherche de la résolution de ses énigmes. Si la mort est presque toujours présente dans cette dernière étape, elle ne constitue cependant pas une fin absolue ; elle constitue plutôt une renaissance, en accord avec une vision surréaliste : La mort n'est qu'une révélation supérieure à la vie. L'errance à laquelle l'absence de tout évènement qui caractérise l'attente est liée, ouvrant sur l'apparition d'une nouvelle expérience souhaitée mais indéterminée, constitue un trait spécifique de l'écriture fictionnelle de Julien Gracq.

\section{BIBLIOGRAPHIE}

ALEXANDRE-GARNER, Corinne \& KELLER-PRIVAT, Isabelle (dir.) (2012). Migrations, Exils, Errances et Écritures. Nanterre : Presses universitaires de Paris Ouest.

BATAILle, Georges (1974). L'Érotisme. Paris : Union Générale d'Édition, « 10/18 », p. 20.

BERTHET, Dominique (dir.) (2007). Figures de l'Errance. Paris : L'Harmattan.

BRETON, André (1988). Second Manifeste du surréalisme, in André Breton, Euvres Complètes, T. I. Paris : Gallimard, Bibliothèque de la Pléiade. 
ERNST, Gilbert (1997). « Entretien sur Un Balcon en forêt », in LEUTRAT, Jean-Louis (dir.) Julien Gracq, Les Cahiers de L'Herne. Paris : Fayard, pp. 209-221.

GLISSANT, Édouard (1997). Traité du Tout-Monde. Paris : Gallimard.

GRACQ, Julien (1989a). Au Château d'Argol, in Julien Gracq, CEuvres Complètes, T. I. Paris : Gallimard, Bibliothèque de la Pléiade.

GRACQ, Julien (1989b). André Breton. Quelques Aspects de l'écrivain, in Julien Gracq, CEuvres Complètes, T. I. Paris : Gallimard, Bibliothèque de la Pléiade.

GRACQ, Julien. Le Rivage des Syrtes (1989c), in Julien Gracq, CEuvres Complètes, T. I. Paris : Gallimard, Bibliothèque de la Pléiade.

GRACQ, Julien (1989d). Préférences, in Julien Gracq, CEuvres Complètes, T. I. Paris : Gallimard, Bibliothèque de la Pléiade.

GRACQ, Julien (1995a). Un Balcon en forêt, in Julien Gracq, CEuvres Complètes, T. II. Paris : Gallimard, Bibliothèque de la Pléiade.

GRACQ, Julien. Lettrines (1995b), in Julien Gracq, Euvres Complètes, T. II. Paris : Gallimard, Bibliothèque de la Pléiade.

GROSSMAN Simone (1980). Julien Gracq et le Surréalisme. Paris : José Corti.

JOURDE, Pierre (1991). Géographies imaginaires. Paris : José Corti.

LAUMONIER, Alexandre (1997). «L'errance ou la pensée du milieu », Le Magazine Littéraire, avril, n 353, pp. 20-25.

LEUTRAT, Jean-Louis. 1967. Julien Gracq. Paris : Editions Universitaires, Classiques du xx siècle. MENOU, Hervé, « Julien Gracq, l'errance du poète », in Bouloumié, Arlette (dir.) (2007). Errance et marginalité dans la littérature. Angers : Presses universitaires de Rennes, pp. 137-148.

MICHON, Pierre (2007). « Une Littérature de l'attente », Entretien avec Pierre Michon, in Le Magazine Littéraire, $\mathrm{n}^{\circ}$ 465, juin, pp. 34-38.

MURAT, Michel (1991). Julien Gracq. Paris : Belfond.

RABOURDIN, Dominique (2007). « Julien Gracq, le dernier des classiques (Dossier) », in Le Magazine Littéraire, nº 465, juin, pp. 24-34.

SEGERS, Marie-Jeanne (2009). De l'Exil à l'errance. ERES.

\section{NOTES}

1. Cet article développe et approfondit quelques aspects effleurés in Ana Isabel Moniz, Julien Gracq : Formas, Sentidos e Mecanismos do Imaginário (2010). Lisboa: Edições Cosmos. 


\section{RÉSUMÉS}

L'errance, thématique récurrente dans l'œuvre de Julien Gracq, présuppose l'envie de nouvelles expériences alliées à un profond désir de changement intérieur. Dans le contexte particulier de sa création littéraire, l'errance est la traduction d'une insatisfaction chez le héros qui le met en quête d'horizons nouveaux. En tant que rupture avec l'autre, en vue d'une radicale transformation de sa vie par transgressions successives, l'errance met en danger l'identité ainsi que l'intégrité d'un sujet qui se dilue pour renaître, transformé et transcendé. Dans cet article, nous nous proposons d'étayer une ou plusieurs réponses aux besoins de cette transformation éprouvés par les êtres fictionnels gracquiens qui les poussent à se déplacer constamment d'un lieu à l'autre, afin de déchiffrer l'énigme du sujet dans sa relation au monde. C'est bien la raison pour laquelle le romancier les fait évoluer dans une perpétuelle oscillation entre le voyage et l'errance.

Wandering, recurrent theme in Julien Gracq's writings, presupposes the will to seek new experiments allied to a profound will of inner change. Within the specific context of his literary creation, wandering unveils a sense of unfulfillment that leads his heroes onto the quest of and discovery of new horizons. As rupture with the past, by means of successive transgressions and continuous ordeals, aiming at a radical change of his life, wandering endangers the identity and integrity of an I that becomes diluted and is reborn transformed. In this article I will try to find one or various explanations to that need felt by Julien Gracq's characters that induce them to constantly roam from place to place aiming at deciphering the enigma of the I in his/her relation with the world. This explains why the author makes them evolve in a constant oscillation between journeying and wandering.

INDEX

Mots-clés : Gracq (Julien), errance, attente, transgression, rupture

Keywords : Gracq (Julien), wandering, the wait, transgression, rupture

\section{AUTEUR}

\section{ANA ISABEL MONIZ}

Universidade da Madeira

anamoniz[at]@uma.pt 